Robotique avancée, intelligence artificielle et développement

\title{
Surveillance collaborative de l'assainissement urbain pour une gestion durable
}

Collaborative monitoring of urban sanitation for sustainable management Monitoramento colaborativo do saneamento urbano para uma gestão sustentável

\section{Abdessalam HIJAB, Hafida BOULEKBACHE et Eric HENRY}

\section{CpenEdition} Journals

Édition électronique

URL : http://journals.openedition.org/ctd/2796

DOI : $10.4000 /$ ctd. 2796

ISSN : 2491-1437

Éditeur

Chaire Unesco Pratiques émergentes en technologies et communication pour le développement

Édition imprimée

ISBN : 2491-1437

Référence électronique

Abdessalam HIJAB, Hafida BOULEKBACHE et Eric HENRY, " Surveillance collaborative de

l'assainissement urbain pour une gestion durable », Communication, technologies et développement [En ligne], 8 | 2020, mis en ligne le 30 juin 2020, consulté le 29 mars 2021. URL : http://

journals.openedition.org/ctd/2796; DOI : https://doi.org/10.4000/ctd.2796

Ce document a été généré automatiquement le 29 mars 2021.

Communication, technologies et développement 


\title{
Surveillance collaborative de l'assainissement urbain pour une gestion durable
}

\author{
Collaborative monitoring of urban sanitation for sustainable management \\ Monitoramento colaborativo do saneamento urbano para uma gestão \\ sustentável
}

Abdessalam HIJAB, Hafida BOULEKBACHE et Eric HENRY

Les actions traditionnelles d'amélioration de la surveillance des réseaux d'assainissement (liquide et solide) en milieu urbain nécessitent, pour le gestionnaire de l'assainissement, un investissement important, humain et technique avec un accroissement des outils de surveillance et des moyens de déplacement in situ, qui à terme se révèlent coûteux.

L'approche que nous avons élaborée a pour objectif d'intégrer le citoyen dans le processus de surveillance du fonctionnement des infrastructures d'assainissement. Nous considérons qu'une forte action contributive citoyenne pour l'amélioration de l'assainissement, aussi bien liquide que solide, concourt aussi à une meilleure protection de l'environnement. Le nom donné à l'approche décrite dans l'article est Géo-CollaboraTIC. Elle associe Systèmes d'Information Géographique (SIG) et Technologies de l'Information et de la Communication (TICs). Pour l'implémenter, nous avons développé une interface interactive et collaborative, en ligne, à référence spatiale. Nous expliquons le principe de fonctionnement de cette technologie qui utilise la géolocalisation en temps réel de données de type topologique et multimédia (photos et vidéos). Cette facilité du monde moderne permet, d'une part, de réduire les coûts de surveillance et de suivi, et d'autre part, de s'emparer des consultations citoyennes du Conseil de l'Europe (communication décisionnelle/citoyen/initiation). La participation des citoyens à la vie de leur cité renforce le dialogue sur le devenir de leur ville et la protection de leur environnement. 


\section{Contexte et problématique}

2 Jusqu'à aujourd'hui, dans les pays en développement ou émergents, la gestion de l'assainissement liquide (eaux usées et pluviales) et solide (déchets de jardinage et de nourriture, déchets de construction et de démolition, déchets industriels, déchets miniers, boues, sacs en plastique, verres, métaux, déchets sanitaires, papiers, vieux vêtements et vieux meubles, etc), est encore loin d'être optimisée.

3 L'assainissement liquide est constitué d'un ensemble de canalisations, de tuyaux et d'ouvrages reliés entre eux pour assurer toutes les opérations de collecte jusqu'au traitement et le rejet. L'ensemble de ces opérations nécessite une bonne maîtrise et connaissance de données multi sources, multi échelles et multi acteurs. L'assainissement solide, en particulier la collecte des déchets ménagers en milieu urbain, oblige les gestionnaires à maintenir des investissements techniques et humains importants.

4 En cas de dysfonctionnement, l'impact sur l'environnement est rapidement perceptible et la qualité du cadre de vie s'en ressent.

5 Plusieurs facteurs conduisent à une difficulté de gestion: en premier lieu, la métropolisation des villes avec ses conséquences dans les domaines de l'habitat, de la santé, de l'environnement, de l'éducation, et, en deuxième lieu, le nombre important de déchèteries et de systèmes d'évacuations des eaux.

6 La volonté d'améliorer la gestion de l'assainissement par une meilleure surveillance des événements liés à un dysfonctionnement se heurte à des coûts difficilement maitrisables et à des résultats pas toujours à la hauteur des attentes.

7 Se pose alors la question du développement de solutions différentes, complémentaires de l'existant, afin d'améliorer la gestion de l'assainissement tout en minimisant les coûts de prise en charge. L'idée d'une prise en charge partagée de la surveillance de l'assainissement, en incitant la remontée d'information par l'habitant, est à explorer. Pour les déchets solides, l'intérêt d'une surveillance de l'assainissement, au plus tôt, au plus près et mieux répartie devrait œuvrer aussi pour une meilleure protection de l'environnement.

De nos jours, la transformation des villes en smart cités offre la mise à disposition constante et rapide de données numériques de toute sorte: multimédias (images, vidéos, schémas...), attributaires (dialogues, textes, chiffres...) et géographiques (cartes, localisations, dessins, plans...). Les derniers développements au niveau des TICs et des SIG ont des conséquences sur le développement psychologique, moral et social des personnes, la structure et le fonctionnement des sociétés, les échanges culturels et la perception des valeurs et des convictions (Douay, $2014: 228$ ).

Depuis 2008, le Conseil de l'Europe promeut l'utilisation des NTIC ${ }^{1}$ pour améliorer la participation des citoyens à la vie de leur collectivité et permettre un dialogue renforcé sur le devenir de leur environnement et de leur cité (Douay, 2014). Par ailleurs, les innovations technologiques en termes d'outils (applications sur Smartphone, réseaux sociaux, site contributif sur wiki, etc.) permettent d'envisager différentes participations et collaborations (Douay, 2011:228; Bailleul, 2008; Bailleul et Gibon, 2013 : 41). Historiquement, (Healey, 1997 ; Bacqué et Gauthier, 2011 : 43 ; Douay, 2014) ont confirmé que l'approche collaborative de planification constitue une dénonciation du modèle traditionnel de planification qui émerge dans la ligne des mouvements 
sociaux et s'inscrit dans l'avènement d'un plus grand pluralisme dans la société qui s'ouvre sur de nouveaux acteurs.

Goodchild (2007:9) a montré également que le citoyen peut être un producteur important d'informations géographiques, et d'autres études pendant la même année de (Kingston et al, $2007: 17)$ ont montré également que le public est devenu très à l'aise avec l'information géolocalisée et les outils de participation électronique, comme les téléphones intelligents et les tablettes.

11 Dans ce contexte, il existe plusieurs approches et applications proposées par des chercheurs :

12 -En 2013, Palskya a proposé une carte participative afin de faire dialoguer des savoirs experts et des savoirs habitants, en utilisant une technique de questionnement et d'entretien destinée à faire émerger des connaissances ou des ressentis à propos des territoires.

13 -La plateforme, Voilà ${ }^{2}$ !portail mobile et web développé en partenariat avec la ville de l'Ancienne-Lorette au Canada, a pour but de faciliter la communication entre les citadins et la ville (intelligente). Un problème non urgent peut être signalé par soumission d'un rapport en ligne, afin d'impliquer les citoyens dans la résolution de problèmes sur leur territoire. Mais cette application ne permet pas de partager l'information géolocalisée sur les réseaux sociaux. Installée sur un Smartphone, elle engendre par ailleurs une consommation de mémoire non négligeable.

14 -Parmi les exemples les plus connus, WikiMapia ${ }^{3}$ est un projet de cartographie participative en ligne, lancé en 2006 par deux informaticiens russes et basé sur l'imagerie satellite de Google et les cartes topographiques. Sur les cartes collaboratives générées, chaque visiteur peut ajouter un lieu en le délimitant par un polygone, puis l'annoter, c'est-à-dire lui associer un titre et un texte descriptif ou un lien, etc. En 2010, plus de 13 millions de lieux étaient identifiés à travers le monde (Palsky, 2010:51). Ce modèle illustre parfaitement le mode participatif de la production des données cartographiques par le public, lequel s'appuie en général sur une plateforme technologique et des processus spatiaux itératifs. Même si WikiMapia est un site web qui contient une base d'informations cartographiques étendue, il ne permet pas d'intégrer les données multimédias.

15 A la lumière de ces travaux de recherche et exemples d'application qui touchent essentiellement aux domaines des TICs, des SIG et des approches dialoguistes participatives, nous avons souhaité proposer une approche qui repose sur l'hypothèse selon laquelle l'association des outils TICs aux SIG favorise l'implication de la population pour une meilleure surveillance des infrastructures de l'assainissement urbain, aspect essentiel de sa bonne gestion.

16 Avant de détailler l'approche que nous avons élaborée, nous faisons un point sur l'usage des TICs et des SIG sur lesquels nous avons porté notre intérêt. 


\section{Le développement des technologies numériques et des SIG}

\section{Les TICs : Technologies numériques pour l'échange d'informations}

Les TICs sont des technologies numériques utilisées dans le traitement et la transmission de l'information, par des supports de plus en plus innovants et mobiles. Cette information joue désormais un rôle crucial dans le fonctionnement des organisations, qu'elles soient publiques, privées ou associatives (Vidal et Desbordes, 2006 : 7). En 2003, l'OCDE ${ }^{4}$ a regroupé les TICs en trois familles: l'informatique, les télécommunications et l'électronique.

$18 \mathrm{Au}$ cours des dernières années, les technologies numériques sont devenues très puissantes et de plus en plus accessibles par toutes les classes sociales, et à tous les niveaux de notre vie quotidienne, notamment Internet. Reprenons les chiffres d'un article $^{5}$ de presse, publié le 7 janvier 2016, sur l'utilisation abondante d'Internet: sur 7,357 milliards de personnes dans le monde, on dénombre 3,715 milliards d'internautes, surtout des jeunes entre 18 et 24 ans, dont le nombre d'utilisateurs représente environ $50,50 \%$ de la population mondiale. Selon Hulime (2009), Internet fait partie intégrante de la vie quotidienne des jeunes. Également plusieurs travaux portant sur l'usage d'Internet soulignent que la consultation des réseaux socionumériques constitue l'activité principale des utilisateurs (Madden et Zickuhr, 2011 ; Salem et al, 2014).

19 Ces technologies ont permis aux internautes de produire leurs propres représentations de l'événement et de ses acteurs, de développer, de partager l'information et des cadres interprétatifs de la mobilisation. Les webcams des Smartphones et des tablettes rendent possibles l'enregistrement de vidéos, la prise de photos et la diffusion en ligne et en temps réel, par les citoyens, via le numérique et les réseaux sociaux (Dymytrova, $2015: 46)$.

Dans un monde de plus en plus numérique, l'usage des TICs devient très important. En effet, les technologies comme Internet, en particulier, favorisent l'acquisition, la transformation et l'échange de l'information, et permettent aux différents utilisateurs d'interagir et de coopérer entre eux. Elles sont considérées comme une chance de dialoguer et de s'exprimer librement en temps réel. Ces technologies d'information peuvent également constituer un instrument solide de protection de valeur, en fournissant l'information qui aide les décideurs à prendre de meilleures décisions ou qui améliore le déroulement des processus. Elles permettent alors aux institutions d'augmenter leurs revenus ou de diminuer leurs coûts (Laudon et al, 2006).

21 Les TICs sont perçues comme des outils de lutte contre la distance et des vecteurs d'une uniformisation du monde (Bakis et al, 2010: 77), idéaux pour mener une opération participative et tendre vers une codécision. Ces technologies se déploient à tous les niveaux de l'activité sociale, culturelle et économique des territoires et sont positionnées au cœur même de leur développement, dans la perspective de consolider le lien entre les citadins et les institutions. Grâce aux TICs, tous les échanges ainsi que tous les partages de connaissances entre les personnes sont devenus possibles sans grandes difficultés (Al Sahyouni Bou Fadel, $2014: 61$ ).

L'accès aux nouveaux outils informatiques et à l'information géographique permet aux citoyens d'exercer la démocratie d'une nouvelle façon. En effet, ils peuvent dorénavant 
participer aux décisions auparavant réservées aux gestionnaires (Pères, 2018:4 ; Mericskay et Roche, 2011).

\section{Les technologies SIG et l'ère GéoWeb}

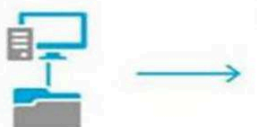

SIG classique

(fichiers géographiques)

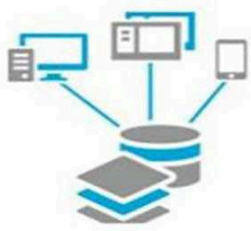

SIG serveurs (bases de données géographiques)

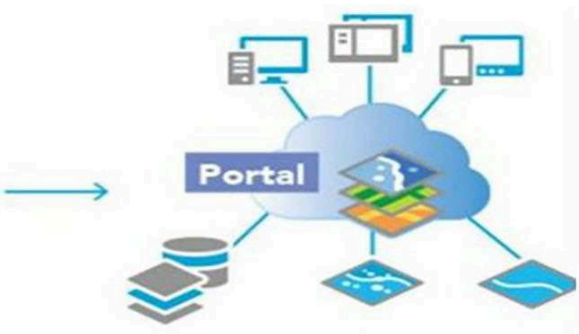

GéoWeb (services Web géographiques)

Figure 1 : Évolution de l'architecture des SIG (ESRI, 2015 ; Mericskay, 2016 : 331) cartes, les annoter et également d'améliorer les actions sur le réseau, en passant de la consultation à l'interaction, ouvrant ainsi l'espace à un nouveau concept d'information géographique volontaire, caractérisé surtout par la mise à jour, parfois instantanée, des contenus en ligne par les internautes (Mericskay et Roche, 2011: 552 ; Pères, 2018: 4 ).

Le GéoWeb ${ }^{6}$ st une nouvelle technologie numérique adaptée à Internet, grand public, orientée vers la géo-visualisation de contenus et la production de cartes personnelles d'une manière rapide et simplifiée. En effet, il vient fondamentalement décloisonner les usages des données cartographiques en s'inscrivant au sein des modes opératoires du Web, par ses nouvelles propriétés d'intégration, de mélange et de diffusion (Mericskay, 
2016: 232). Ce type de cartographie intéresse un nombre croissant de chercheurs (géographes, agronomes, urbanistes, anthropologues, etc) et des consultants de différentes institutions (locales ou internationales), ayant pour objectif de faire participer les communautés locales à la représentation du lieu qu'elles habitent et d'en dégager les éléments utiles pour leur implication dans les actions territoriales. Le GéoWeb est reconnu comme un instrument qui facilite le dialogue d'une pluralité d'acteurs, pour leur permettre de conduire une négociation ayant pour objet commun le territoire habité par une communauté qui y a déposé au cours des années leurs propres valeurs et savoirs (Burini, $2008: 1$ ).

Grâce au développement important d'outils SIG libres, du Web Mapping (Mymaps Google, ArcGIS online ${ }^{7} . .$. ), des TIC (Internet, Smartphones, Tablettes, réseau sociaux...) et du GPS, de nouvelles opportunités dans la diffusion plus large de l'information géolocalisée, s'offrent aux décideurs et aux citadins en favorisant un canal de communication, d'interactivité et de rapprochement entre eux dans plusieurs domaines, notamment l'assainissement urbain (secteur d'application de notre recherche).

\section{La Géo-CollaboraTIC, une approche au service de la surveillance de l'assainissement urbain pour une gestion durable}

\section{Objectif et schéma de principe}

L'analyse des travaux de recherche, des usages et des retours d'expérience en lien avec l'utilisation par la population des TICs et des SIG a consolidé notre objectif d'élaborer une approche pour impliquer les habitants dans le processus de surveillance de l'assainissement liquide et solide, à l'aide de ces deux technologies.

L'intérêt des TICs est qu'elles sont omniprésentes dans la population et d'utilisation intuitive ; l'avantage des SIG est que leurs applications sont de plus en plus répandues et d'utilisation de plus en plus simplifiée et mobile. Le couplage de ces deux technologies et leur mise en œuvre sont à coûts modérés et rapidement fonctionnels.

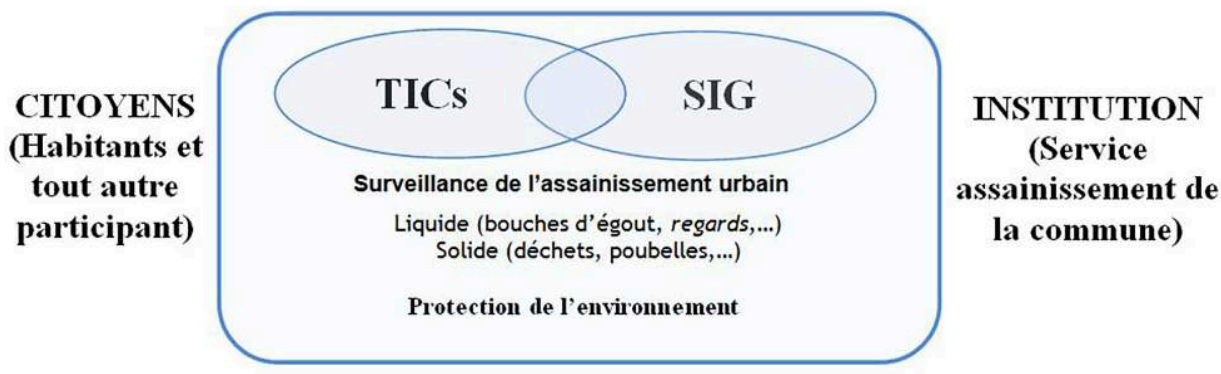

Figure 2 : La Géo-CollaboraTIC au service de l'assainissement et de la protection de l'environnement

31 Nous avons rendu ce couplage fonctionnel en élaborant une approche que nous avons nommée « Géo-CollaboraTIC ». La « figure 2 » en donne une schématique. 
32 Nous l'avons rendu opérationnel en développant une plateforme afin de répondre aux besoins du service d'assainissement d'un quartier de Casablanca. Dans les pages qui suivent, nous nous servirons de la problématique de ce cas d'étude pour expliquer l'approche Géo-CollaboraTIC et pour illustrer le processus d'association des TICs aux outils SIG d'une collectivité. Le cas d'étude fera l'objet d'une présentation plus précise un peu plus loin.

\section{Les fonctionnalités de la Géo-CollaboraTIC}

33 L'approche Géo-CollaboraTIC, telle que nous l'avons imaginée suit un processus en deux étapes, illustrées sur la «figure 3 »:

34 - la première est l'identification des problèmes observés par les habitants eux-mêmes par le biais d'un TIC. En faisant appel à la cartographie en ligne, affichable sur son appareil (SIG), l'habitant envoie un message d'alerte géolocalisée au service d'assainissement sous forme d'une photo, d'une vidéo, d'un texte descriptif ou les trois à la fois ;

35 - la deuxième étape est la transmission du message géolocalisé au gestionnaire de l'assainissement urbain et son traitement. Ce message qui contient une alerte ${ }^{8}$ est lu, archivé et traité par le service technique. Après la réception du message, l'analyse et la prise en charge du problème sur le terrain, une notification de remerciement est envoyée à l'envoyeur collaborateur.

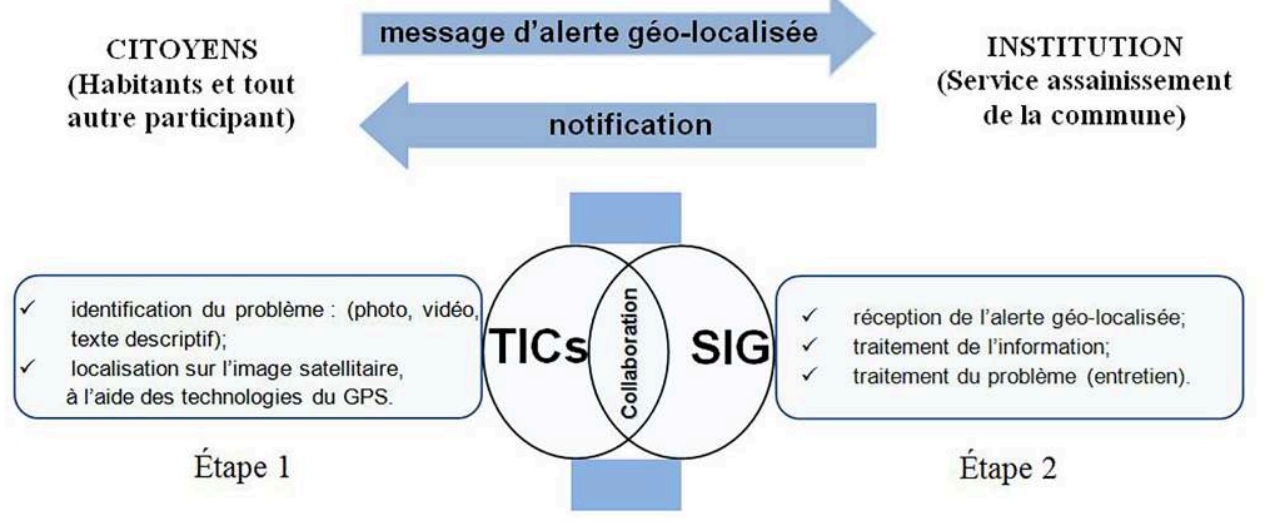

Figure 3 : Descriptif de l'approche Géo-CollaboraTIC

Ainsi, le traitement de d'information entre les habitants collaborateurs et le service de gestion de l'assainissement urbain, se caractérise par deux types de communication selon la schématisation systémique de l'approche « figure 4 »:

- une communication externe consistant en un échange d'informations sous forme d'envoi de messages d'alertes renseignées et géolocalisées par les citoyens au gestionnaire et, en retour, d'envoi de notifications de bonne réception par le gestionnaire vers les habitants contributeurs ;

38 - une communication interne entre les acteurs internes du service de gestion (les agents de bureau et les agents de terrain), ce qui devrait se traduire par une intervention adaptée sur site. 


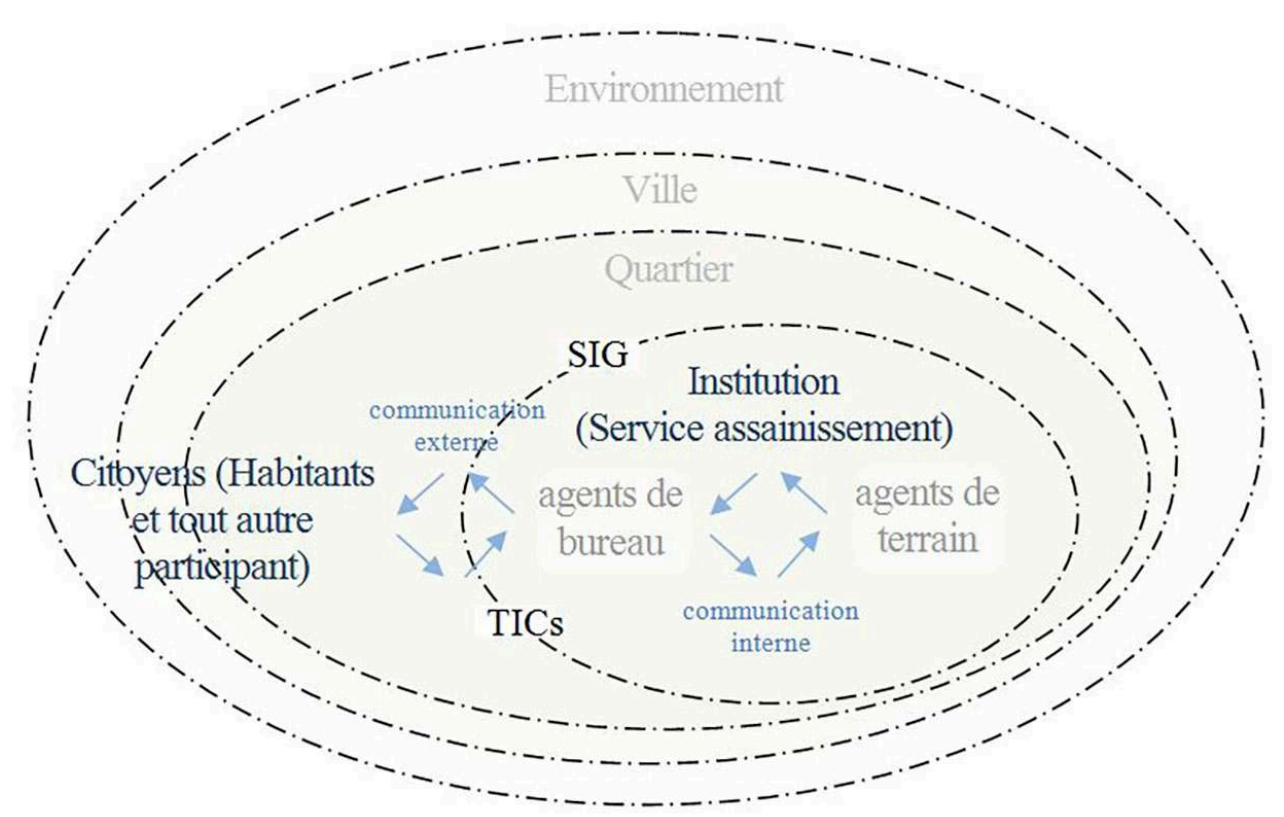

Figure 4 : Schématisation globale de la Géo-CollaboraTICet type de communication mise en œuvre

$$
\begin{aligned}
& \text { utilisant les supports de la cartographie participative et de la géolocalisation en ligne } \\
& \text { des données. }
\end{aligned}
$$

\section{Mise en œuvre d'une plateforme}

Pour pouvoir implémenter l'approche Géo-CollaboraTIC, il a été nécessaire de mettre en œuvre une plateforme constituée de TICs et de SIG, interconnectés par le biais d'un lien généré par une application mobile GeoWeb dont la cartographie a été enrichie par la base de données géographiques de la collectivité. Le lien est fourni par la collectivité aux citadins par le biais d'un mail d'information ou à charger sur le site internet de la commune.

La plateforme a été baptisée «Géo-Protect-L ». Les TICs sont utilisées comme points d'entrée de l'information et comme moyens de saisie simples, intuitifs, opportunistes, bien répartis sur le territoire et principalement dans les lieux de passage. Le citadin va contribuer ainsi à une surveillance maillée des dysfonctionnements du système d'assainissement, par la remontée des problèmes palpables «figure 5 » et leur localisation précise dans le système de repérage du service d'assainissement, défini par le SIG créé et développé en son sein.

La TIC préférentielle choisie est de type Smartphone ou tablette connectée. Par le biais de l'application mobile, l'utilisateur peut saisir le problème à remonter parmi une liste à choix multiple, et indiquer l'information de géolocalisation. 


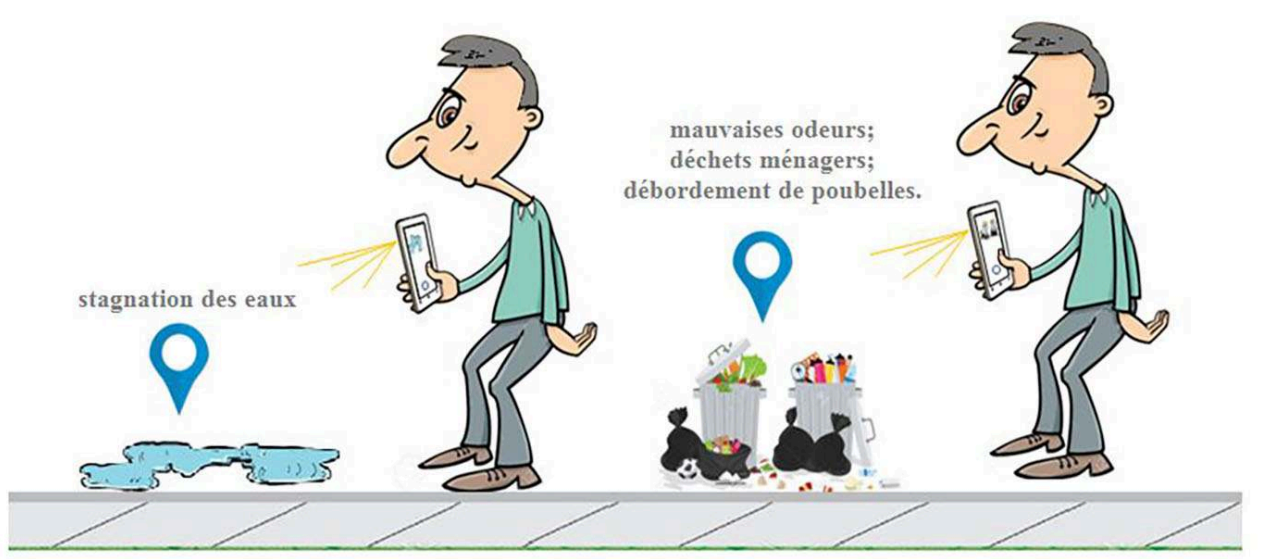

Figure 5 : Signalisation des problèmes à l'aide des alertes géolocalisées envoyées par le citadin

Pour simplifier la saisie, la liste à choix multiple contient les informations de type : " présence de déchets ", " débordement des égouts ", " perte de tampon de regard", « fuites », « stagnation des eaux », « présence des mauvaises odeurs », etc.

La création de la plateforme Géo-Protect-L propre à la collectivité a nécessité d'ouvrir un compte de type Google Mymaps ${ }^{9}$ pour accéder à la cartographie en ligne de la localité, d'y insérer le tracé du schéma d'assainissement en format $\mathrm{KMZ}^{10}$, et enfin de le partager plus largement par un lien via les TICs retenues.

L'application mobile Google Mymaps permet de créer et d'ajouter des dessins (points, lignes et formes), d'insérer des images, des photos, des vidéos et de les compléter par des informations descriptives, des suggestions, liées aux dysfonctionnements signalés. La possibilité d'enrichir l'information de base de l'application, de la traiter par le service gestionnaire et, surtout, d'en faire un feedback, valorisent l'implication du citoyen contributeur. Cela devrait l'encourager à poursuivre les échanges au bénéfice de la protection de l'environnement.

\section{Application pour le service d'assainissement du quartier de Lamkansa}

Nous avons expérimenté l'approche Géo-CollaboraTIC auprès $\mathrm{du}$ service d'assainissement du quartier Lamkansa «Figure 6 ». Il a été choisi comme quartier test car il est représentatif des problèmes d'état et de gestion de l'assainissement de nombreux quartiers. 


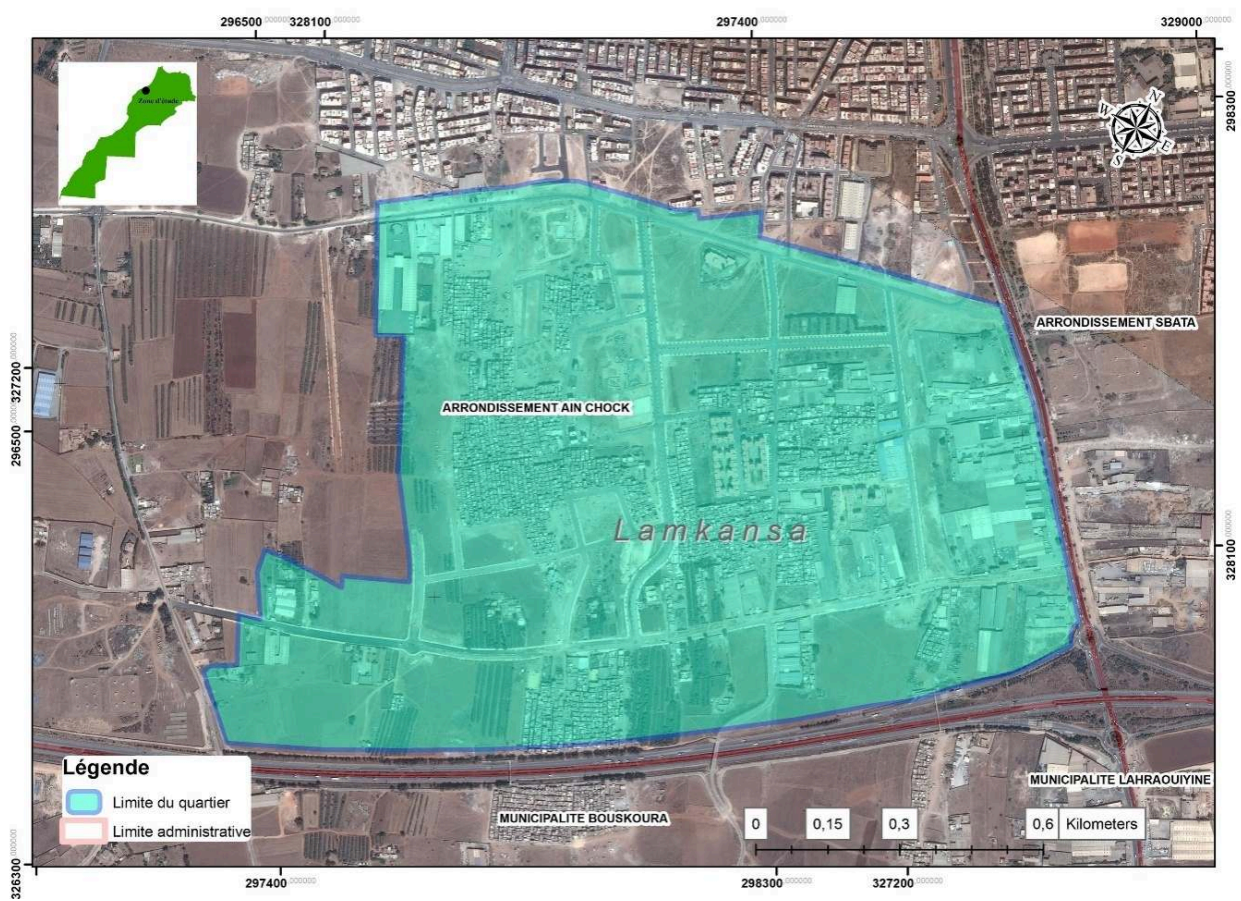

Figure 6 : Vue spatiale du quartier Lamkansa

Casablanca au Maroc, d'une superficie d'environ 300 ha, et sa population en 2014 est estimée par LYDEC $^{11}$ à 32000. Dans ce quartier, le réseau d'assainissement existant présente des risques majeurs. À partir d'une vue satellitaire «Figure 3 » et d'un contrôle de terrain, on observe une occupation anarchique de l'espace urbain caractérisée par une insuffisance et une disparité spatiale des infrastructures et des problèmes d'assainissement liquide et solide. Dans (Hijab et al, 2018; Hijab et Boulekbache, 2018) nous donnons un aperçu de l'environnement dégradé du quartier.

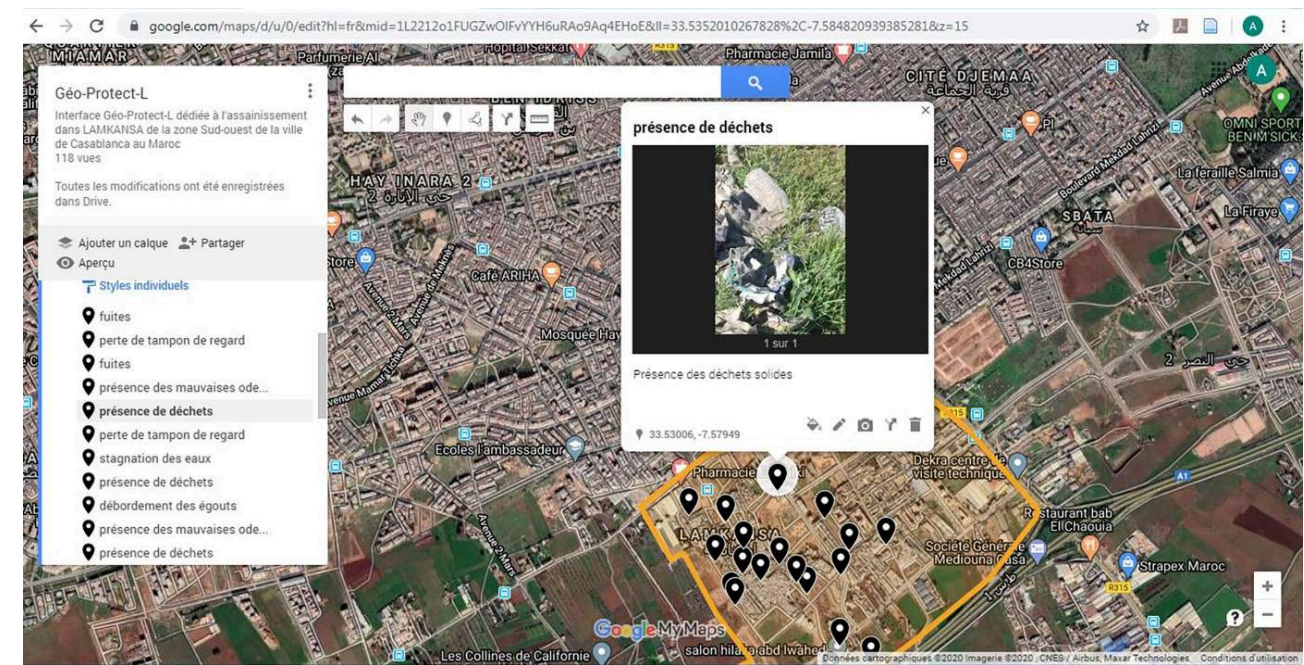

Vue a/ Alerte saisie et partagée par un habitant 


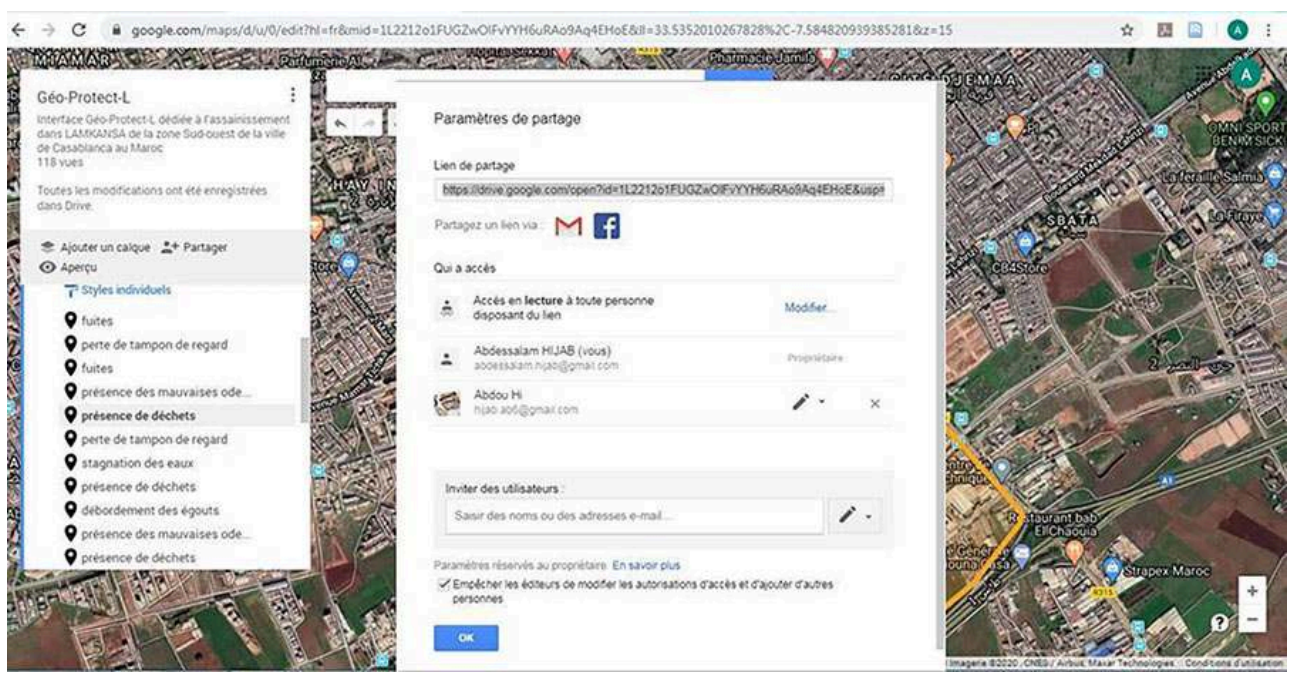

Vue b/ Réception de l'alerte et traitement du problème au sein du service assainissement

Figure 7 : Vues d'écran de la plateforme Géo-Protect-L sur MyMaps-Google «quartier Lamkansa»

Via l'interface de la plateforme interactive à référence spatiale Géo-Protect-L visible sur la " figure 7 », les citadins peuvent recommander, géolocaliser et envoyer des alertes au service assainissement (Vue a) pour analyse, traitement et intervention sur place (Vue b). Ce dispositif pourra à terme alimenter un système de capitalisation des données (alphanumériques et cartographiques) et une archive utile pour des analyses de gestion et d'aide à la décision. Ainsi, la représentation spatiale des données capitalisées sous forme de cartes thématiques améliore l'identification des zones de risques, l'analyse des causes et la simulation des phénomènes.

\section{Promotion et développement futur}

Pour sensibiliser la population, il est envisagé de faire de la pédagogie sur l'intérêt de s'investir par le biais d'encart dans les journaux et dans les réseaux sociaux, et de distribuer des flyers promotionnels. Pour l'encourager à participer, des points d'information seront mis en place au carrefour des zones d'habitation (stationnement de caravanes). Pour une incitation encore plus forte, un système de capitalisation de points associé à des récompenses (obtention d'une somme d'argent ou de coupons d'achats, etc) pourrait être implanté dans l'application.

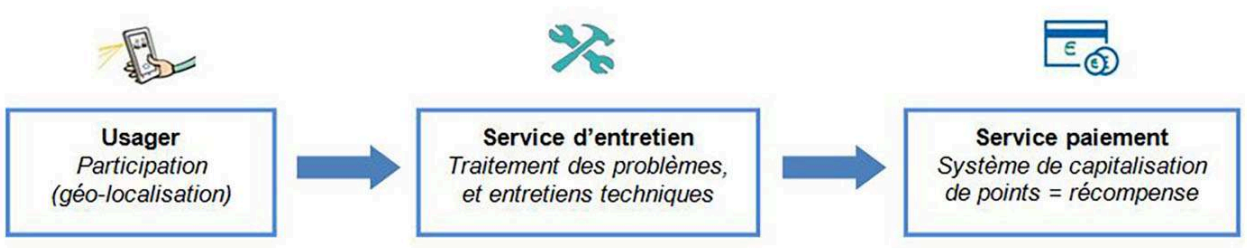


Figure 8 : Système de capitalisation de points proposé pour motiver les habitants à participer

50 Dans ce présent travail, nous nous sommes limités à utiliser des techniques incitatives basées sur la publicité et la pédagogie. Le système de capitalisation de points « figure 8 » sera intégré ultérieurement.

51 Ce développement futur nous parait incontournable pour deux raisons :

52 - l'implication forte des habitants au sein de leur quartier laisse présager une limitation des investissements techniques et humains pour lesquels la tendance est à l'augmentation en raison d'exigence accrue en matière d'élimination des déchets et de protection de l'environnement ;

53 -la mise en œuvre d'une collaboration soutenue avec la population tend à réduire le temps de diagnostic des dysfonctionnements. Une alerte déclenchée, le plus tôt possible et bien géolocalisée, amène à produire un plan de circulation précis en économisant temps et énergies pour traiter le problème, par les agents de terrains.

54 Enfin, pour faciliter l'accès à la plateforme Géo-Protect-L, nous envisageons le développement d'une application mobile, plus dans l'air du temps, téléchargeable que nous pourrions nommer Smart-Protect-L.

\section{Les atouts de la Géo-CollaboraTIC}

55 Grâce à l'intégration de la population dans les processus de suivi, de surveillance et de communication décisionnelle, l'approche Géo-CollaboraTIC amène à réduire les coûts de surveillance de l'assainissement urbain, la rend plus efficace et implique des interventions plus rapides pour une gestion plus durable.

Les points forts de l'approche proposée sont :

57 - une plus grande sensibilisation de la population au respect de son environnement ;

58 - l'intégration du citoyen au développement durable de sa ville en l'encourageant psychiquement à devenir un habitant participatif, actif et dynamique ;

59 - une incitation plus forte des décideurs à davantage impliquer les habitants par le biais de l'utilisation couplée des technologies SIG/TICs, non seulement pour la surveillance de l'assainissement, mais aussi en matière de planification urbaine ;

60 - l'utilisation de TICs disponibles et répandues dans la population, de manipulations devenues intuitives, sans générer de surcoût, cela en faveur d'un meilleur fonctionnement et embellissement de la ville ;

61 - la localisation exacte des problèmes (à l'aide de la technologie GPS intégrée dans les Smartphones et tablettes, etc), ce qui minimisera les temps d'entretien et enrichira une base de données à référence spatiale déjà existante ;

62 - l'établissement de cartes d'analyse des points noirs, d'anomalies, de données hors norme, etc ;

63 - des procédés faciles à mettre en œuvre pour transformer l'habitant en smart habitant, comme élément pilier pour une smart ville. 


\section{Bilan et perspective}

\section{BIBLIOGRAPHIE}

\section{Bibliographie}

Al Sahyouni Bou Fadel, R. (2014). TIC et apprentissage de l'interculturalité. Doctoral dissertation, Bordeaux 3. https://tel.archives-ouvertes.fr/tel-01143443/document (source consultée le 04 juin 2019).

Bacqué, M. H., \& Gauthier, M. (2011). Participation, urbanisme et études urbaines. Quatre décennies de débats et d'expériences depuis «A ladder of citizen participation » de S. R. Arnstein, in Participations, pp. 36-66. https://www.cairn.info/revue-participations-2011-1page-36.html \%23top (source consultée le 04 juin 2019).

Bailleul, H. (2008). Les nouvelles formes de la communication autour des projets urbains : modalités, impacts, enjeux pour un débat participatif. Métropoles 3. http:// metropoles.revues.org/2202 (source consultée le 05 juin 2019).

Communication, technologies et développement, 8 | 2020 
Bailleul, H., \&Gibon, C. (2013). Médiation urbaine numérique Quels enseignements pour un urbanisme participatif? in Place Publique, $n^{\circ} 25$ (dossier : Faut-il avoir peur de la ville numérique ?), pp. 39-44. http://www.placepublique-rennes.com/media_site/upload/PP25DOSSIER.pdf (source consultée le 05 juin 2019).

Bakis, H., \& Vidal, P. (2010). Geography of the Information Society, in B. Reber \& C. Brossaud (eds.), Digital Cognitive Technologies. Epistemology and Knowledge Society, ISTE / Wiley, Ch. 5, pp. 71-87.

Burini, F. (2008, juin). La cartographie participative et la pratique du terrain dans la coopération environnementale : la restitution des savoirs traditionnels des villages de l'Afrique subsaharienne. In À travers l'espace de la méthode : les dimensions du terrain en géographie, Communication au colloque "À travers l'espace de la méthode : les dimensions du terrain en géographie", Arras. https://halshs.archives-ouvertes.fr/halshs-00389595/document (source consultée le 05 juin 2019).

Didier Michel. (1990). Utilité et valeur de l'information géographique, Paris, Economica, STU, CNIG. $255 \mathrm{p}$.

Douay, N. (2011). Urbanisme 2.0, de la contestation à la participation. In TechniCités, n²14 (supplément spécial pour la 32ème rencontre nationale des agences d'urbanisme, Territoires et Projets. Les outils de la gouvernance), pp. 46-49.

Douay, N. (2014). Les usages du numérique dans le débat public. Devenirs urbains, Collection Territoires numériques, Paris : Presses des Mines, 227-244. http://www.grico.fr/wp-content/ uploads/2017/03/Devenirs-urbains-DOUAY-V.pdf (source consultée le 04 juin 2019).

Dymytrova, V. (2015). Les nouvelles technologies numériques au service d'une mobilisation collective : le cas de la Révolution de la dignité en Ukraine. Communication, Technologies et Développement 2, pp. 42-52. http://www.comtecdev.com/fr/telecharger/Dymytrova.pdf (source consultée le 06 juin 2019).

Feyt, G. (2004). Les métiers du territoire face aux technologies de l'information géographique : Babel et esperanto. In Roche S., Carron C. (éd.), Aspects organisationnel des SIG, Paris : Hermès Lavoisier, pp. 55-70.

Gale, G. (2013). Push pins, dots, customisation, brands and services : the three waves of making digital maps, The Cartographic Journal, $\mathrm{n}^{\circ}$ 50, p. 155-160.

Goodchild, M. F. (2007). Citizens as sensors : web 2.0 and the volunteering of geographic information. GeoFocus. Revista Internacional de Ciencia y Tecnología de la Información Geográfica, (7), 8-10.

Healey, P. (1997). Collaborative Planning, Shaping Places in Fragmented Societies. Vancouver, University of British Columbia Press.

Hijab, A and Boulekbache, H. (2018). Design and sustainable management of liquid wastewater treatment network using the GIS tool and the space-based database (Geo-LD).40th IABSE Symposium Nantes 2018, tomorrow's megastructur's, Nantes, France, ISBN : 978-3-85748-153-6, pp. S24-1-S24-8.

Hijab, A, Boulekbache, H et Henry, E. (2018, septembre). L'information géolocalisée au service de l'évaluation technico-spatiale du réseau d'assainissement des eaux pluviales et de la protection de l'environnement (Sud-ouest de Casablanca). Communication présentée à la $71^{\mathrm{e}}$ conférence canadienne de géotechnique et la 13e conférence conjointe SCG/AIH-SNC sur les eaux souterraines, GéoEdmonton 2018, Edmonton, Alberta, Canada. 
Hulme, M. (2008). Life Support : Young people's needs in a digital age. Youth Net report.

Kingston, R, Carver, S, Evans, A., \&Turton, I. (2007). Web-based public participation geographical information systems : an aid to local environmental decision-making, Centre for Computational Geography, School of Geography, University of Leeds, U.K.,17p.

Laudon, K. and J. Laudon. (2006). Management Information Systems : Managing the Digital Firm, 9th Edn., Prentice Hall, (country).

Laurini, R., \&Milleret-Raffort, F. (1993). Les bases de données en géomatique. Paris, Hermès ; $340 \mathrm{p}$.

Longley P.A., Goodchild M.F, Maguire D.J., \&Rhind D.W. (2005).Geographical Information System : Principles, Techniques ; Management and Applications (abridge edition). Hoboken, N.J. : Wiley, 404p.

Madden, M. and Zickuhr, K. (2011). 65 \% of online adults use social networking sites. Washigton, D. $C$ : Pew Internet and American Life Project.

Mericskay, B. (2016). La cartographie à l'heure du Géoweb : Retour sur les nouveaux modes de représentation spatiale des données numériques. Cartes \& géomatique, 229, 37-50. https:// halshs.archives-ouvertes.fr/halshs-01468314/ (source consultée le 05 juin 2019).

Mericskay, B., \& Roche, S. (2011).Cartographie 2.0 : le grand public, producteur de contenus et de savoirs géographiques avec le Web 2.0, Cybergeo : European Journal of Geography, 552. https:// journals.openedition.org/cybergeo/24710 (source consultée le 05 juin 2019).

Palsky, G. (2010). Cartes participatives, cartes collaboratives. La cartographie comme maïeutique - Le Comité Français de Cartographie (CFC), Paris, n. 205, p. 49-59.

Palsky, G. (2013). Cartographie participative, cartographie indisciplinée. L'Information géographique, 77(4), 10-25.

Pères, C. A. (2018). Choix et analyse d'un système d'information géographique interactif pour l'aide à la gestion urbaine et la participation citoyenne. Doctoral dissertation, Université de Sherbrooke.

Ratcliff, E. (2007). Google Maps is changing the way we see the world. Wired Magazine, $\mathrm{n}^{\circ} 15$.

Salem, F., Mourtada, R., \&Alshaer, S. (2014). The Arab World Online 2014 : Trends in Internet and Mobile Usage in the Arab Region. Dubai : Mohammed Bin Rashid School of Government.

Vidal, P., \& Desbordes, F. (2006). Les technologies de la société de l'information (TIC/SIG) au service de l'aménagement et du développement des territoires en Afrique de l'Ouest. ENEA Dakar/Université Toulouse Le Mirail.

\section{NOTES}

1. Le terme qui a été utilisé pour des technologies de l'information et de la communication, par Conseil de l'Europe en 2008.

2. http://www.appvoila.com/fr/\#about (source vérifiée le 04 avril 2020).

3. http://blog.cartong.org/2014/07/21/tuto-utiliser-la-donnee-wikimapia-dans-qgis/ (source vérifiée le 04 avril 2020).

4. Organisation de Coopération et de Développement Économiques : organisme situé à Paris, compte 35 pays membres et regroupe plusieurs centaines d'experts depuis 2010. Elle publie fréquemment des études économiques - analyses, prévisions et recommandations de politique 
économique - et des statistiques, principalement concernant ses pays membres (wikipédia, source vérifiée le 04 avril 2020).

5. Article de presse, publié par Thomas Coëffé, https://www.blogdumoderateur.com/50-chiffresmedias-sociaux-2016/ (source vérifiée le 04 avril 2020).

6. Nouveau régime cartographique grand public, basé sur la convergence des technologies SIG et du TICs. En effet, d'une manière générale se sont les applications géographiques accessibles via l'internet.

7. Composant clé et fait partie intégrante du système ArcGIS d'Esri. C'est un système de gestion de contenu composé d'applications et de modèles pour créer des cartes interactives (Wikipédia, source vérifiée le 04 avril 2020).

8. Sous forme de photos, vidéos et/ou texte descriptif, ces alertes sont géolocalisées sur image satellitaire, à l'aide de SIG en ligne et technologies GPS sur les Smartphones et tablettes.

9. Service lance par Google en mai 2007, qui permet aux utilisateurs de créer des cartes personnalisées à usage personnel ou de partage.

10. Le KMZ est un fichier KML compressé (Keyhole Markup Language; en français : langage à base de balises géo locales), est un ensemble de caractéristiques (lieu des marques, des images, des polygones, des modèles 3D, des descriptions textuelles, etc.) pour l'affichage en ligne dans Here Maps, Google Earth, Maps et Mobile, ou tout autre logiciel géospatial (Wikipédia, source vérifiée le 04 avril 2020).

11. Lyonnais des eaux de la ville de Casablanca. Entreprise qui gère l'eau, l'électricité et l'assainissement dans la ville de Casablanca.

\section{RÉSUMÉS}

Traditional improvement actions for monitoring sewerage networks (liquid and solid) in urban areas require a significant human and technical investment from the urban sanitation administration, due to the increase in costs of monitoring tools and means of transport on-site in the long run. The approach we developed aims to propose integration of the citizen in the process of monitoring the operation of sanitation infrastructures. We consider that a strong citizen contribution to the improvement of sanitation, both liquid and solid, also contributes to a better protection of the environment. The name given to the approach described in the article is Geo-CollaboraTIC. It combines Geographic Information Systems (GIS) and Information \& Communication Technologies (ICT). To implement it, we developed an interactive and collaborative online spatially referenced interface. We explained the operating principle of this technology, which uses real-time geo-localization of topological and multimedia data (photos and videos). Such facilities of the modern world make possible, on the one hand, to reduce surveillance and monitoring costs, and on the other hand, to take over the Council of Europe's citizen consultations (decisional communication/citizen/initiation). The participation of citizens in the life of their city strengthens the dialogue about its future and the protection of their environment.

As tradicionais ações de melhoria para o monitoramento das redes de esgoto, líquido e sólido, em áreas urbanas exigem um investimento significativo da administração de saneamento urbano para recursos humanos e técnicos devido ao alto custo das ferramentas de monitoramento e dos meios de deslocamento in situ em longo prazo. A abordagem que desenvolvemos visa propor a 
integração do cidadão no processo de monitoramento das infraestruturas de saneamento. Consideramos que ao contribuir para a melhoria do saneamento, tanto líquido como sólido, o cidadão também contribui para uma melhor proteção do meio ambiente. O nome dado à abordagem descrita no artigo é Geo-CollaboraTIC. Ela combina Sistemas de Informação Geográfica (SIG) e Tecnologias de Informação e Comunicação (TIC). Para a sua implementação, desenvolvemos uma interface online interativa e colaborativa, de referência espacial. Este trabalho explica o princípio de operação desta tecnologia, que utiliza a geo-localização em tempo real de dados topológicos e multimídia, como fotos e vídeos. Estas facilidades do mundo moderno permitem reduzir os custos de vigilância e monitorização, bem como envolver as consultas dos cidadãos conforme o Conselho da Europa (comunicação/cidadão/iniciativa decisória). A participação dos cidadãos na vida da sua cidade reforça o diálogo sobre o seu futuro e a proteção do seu ambiente

\section{INDEX}

Mots-clés : SIG, TICs, Géo-CollaboraTIC, Citadin, réseau d'assainissement.

Keywords : GIS, ICT, Geo-CollaboraTIC, citizen, sanitation network.

Palabras claves : GIS, TIC, Geo-CollaboraTIC, cidadão, rede de saneamento.

\section{AUTEURS}

\section{ABDESSALAM HIJAB}

Univ. Polytechnique Hauts-de-France, EA 244 DeVisu - Laboratoire en Design Visuel et Urbain, F-59313 Valenciennes, France

\section{HAFIDA BOULEKBACHE}

Univ. Polytechnique Hauts-de-France, EA 244 DeVisu - Laboratoire en Design Visuel et Urbain, F-59313 Valenciennes, France

\section{ERIC HENRY}

Univ. Polytechnique Hauts-de-France, EA 244 DeVisu - La

boratoire en Design Visuel et Urbain, F-59313 Valenciennes, France. 